\title{
Aluminum Sintering in Air Atmosphere Using High Frequency Induction Heating
}

\author{
J.M. Mendoza-Duarte ${ }^{1}$, F.C. Robles-Hernandez ${ }^{2}$, I. Estrada-Guel ${ }^{1}$, R. Martínez-Sánchez ${ }^{1}$ \\ 1. Centro de Investigación en Materiales Avanzados (CIMAV). Laboratorio Nacional de Nanotecnología \\ Miguel de Cervantes No. 120, 31136, Chihuahua, Chih., México. \\ 2. Department of Mechanical Engineering Technology, University of Houston, Houston, TX 77204- \\ 4020, USA.
}

For decades, aluminum (Al) has been the most widely used industrial metal (after steel) for its appreciated properties. Unfortunately, the pure metal presents a lower mechanical response; normally to counter this disadvantage some alloy elements are added. However, its high electrical-thermal conductivity and corrosion resistance are compromised. As an alternative, the cold working process can be used to increase mechanical performance, but the ductility is drastically reduced due matrix embrittlement (dislocations saturation). Another hardening route is based on grain refinement (at submicron or nanometric level), where the material properties are positively modified. The mechanical milling (MM) technique involves repeated impacts between the sample and the grinding media causing plastic deformation, fracture and cold welding reaching a highly refined microstructure. After MM, some compaction and heat treatment steps are applied to milled powders to obtain solid samples. However, during the sintering process long term heating promotes a remarkable grain growth, destroying the highly refined state reached after MM.

The present work deals with the preparation of some microstructural refined Al samples and sintered by high frequency induction heating (HFIS). Using HFIS is possible to process materials in minutes instead hours, reaching high densification levels with low costs (investment and processing). Refined Al powders were prepared using a SPEX-800M mixer/mill during 2 hours in a hardened steel vial. For comparison proposes, milled powders were sintered using two routes: a) conventional sintering (CS) and HFIS. With CS the samples were cold compacted under $900 \mathrm{MPa}$ pressure and then sintered at $550^{\circ} \mathrm{C}$ using a heating rate of $10^{\circ} \mathrm{C} / \mathrm{min}$ for 3 hours and b) HFIS, where the samples were compacted and sintered $\left(450 \mathrm{MPa}-450^{\circ} \mathrm{C}\right)$ at the same time for 3 minutes, following a heating slope of $158^{\circ} \mathrm{C} / \mathrm{min}$. In order to simplify the HFIS process, it was carried out in an air atmosphere.

The figure 1 shows the XRD patterns of milled and sintered Al samples. With milling, Al peaks become broader and their intensity decreases (Al-0h and Al-2h samples); this is related with severe plastic deformation and grain refinement. After sintering, there is no shift or presence of new peaks suggesting low contamination or alumina formation during sintering (specially the HFIS samples processed under an air atmosphere). Furthermore, we can notice an important grain size growth during CS meanwhile HFIS maintains the refined microstructure.

Figure 2 exhibit some EDS analyses performed on the surface of sintered samples showing a low concentration of oxygen in comparison with CS sample prepared under an inert Ar Atmosphere and cold compacted green sample.

Based on previous evidence, induction heating proved that is a convenient and practical method for aluminum sintering. HFIS processed samples show low oxygen contamination and grain size retention due its low temperature and short processing time. 


\section{References:}

[1] S. Hurley, Met. Bull. Mon. (1995) 54-56.

[2] M. Raviathul Basariya, V.C. Srivastava, N.K. Mukhopadhyay. Materials and Design 64 (2014) 542549.

[3] Jong-Yeol Yoo, In-Jin Shon, Byung-Hyun Choi, Ki-Tae Lee. Ceramics International 37 (2011) 2569-2574.

[4] In-Jin Shon, In-Kyoon Jeong, In-Yong Ko, Jung-Mann Doh, Kee-Do Woo. Ceramics International 35 (2009) 339-344.

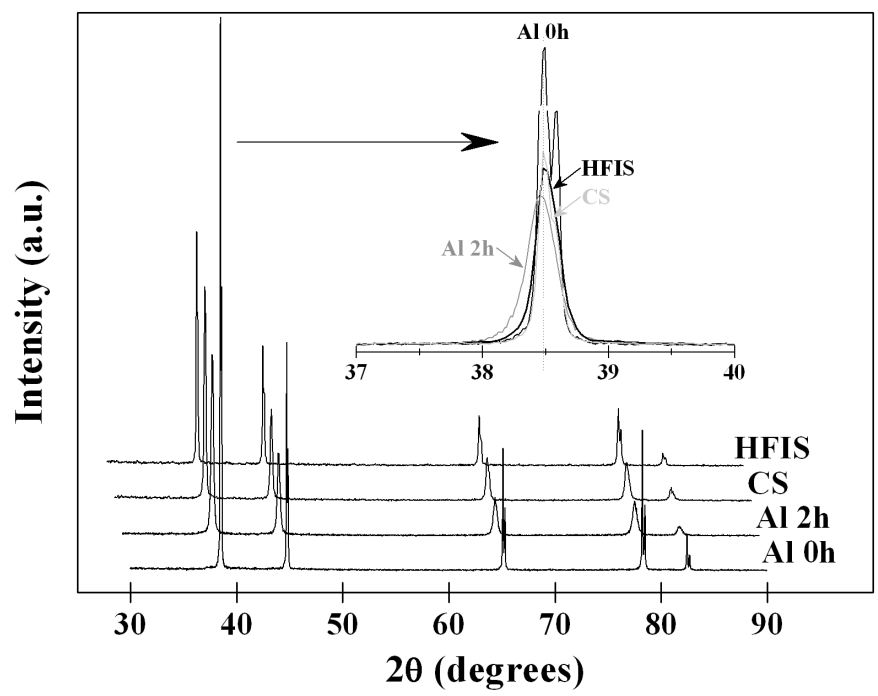

Fig. 1. XRD patterns with a close up on Al (111) peak of studied samples: green (Al-0h), milled (Al-2h), conventional sintered (CS) and HFIS.
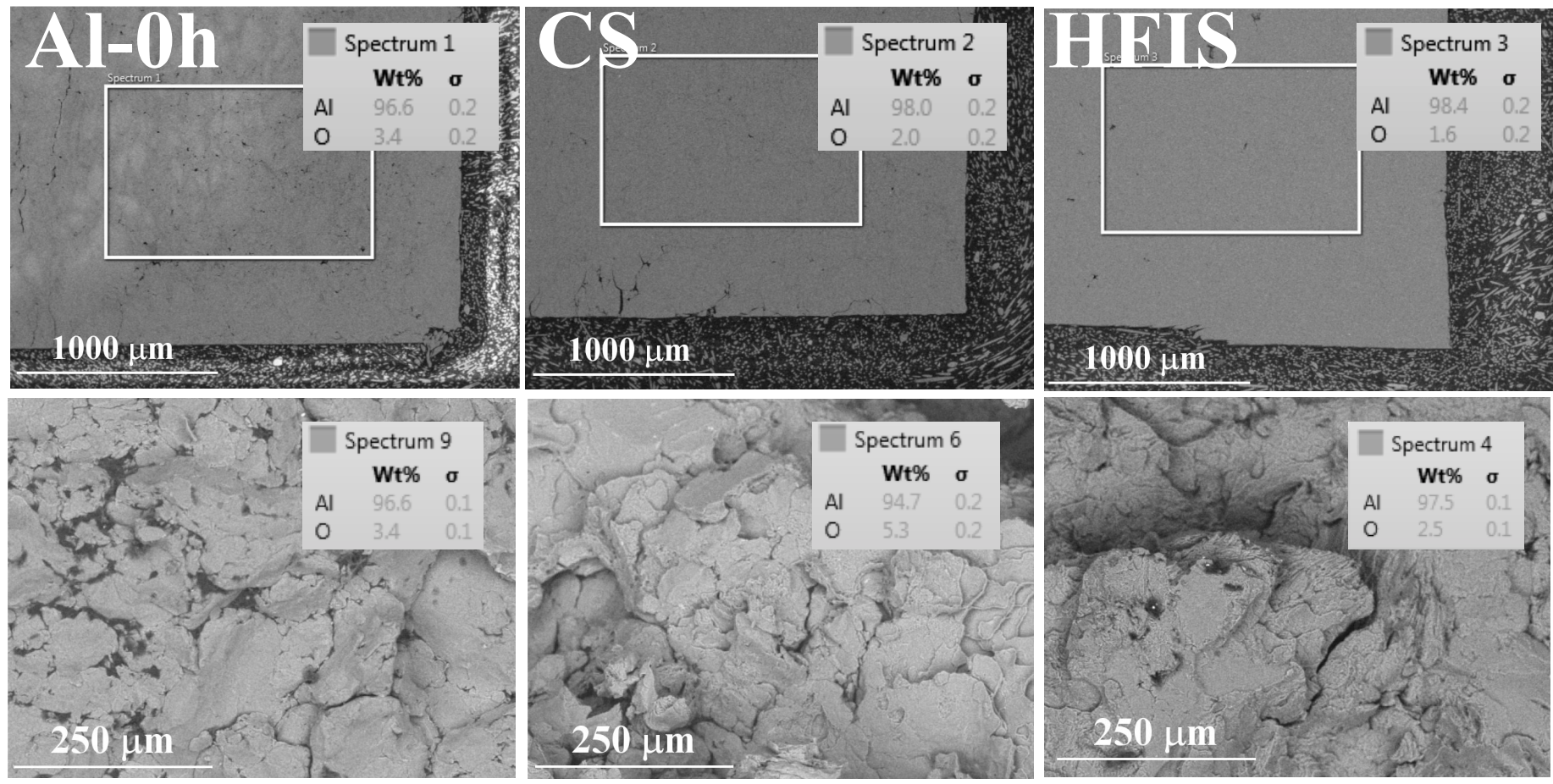

Fig. 2. SEM images of Al samples and EDS results on limited area (white square) and general zone (below). Note the oxygen concentration of samples, although HFIS samples were processed in Air, these showed the lowest O contamination level. 